\title{
Simulation of viscous melt drop crystallization by the SPH method
}

\author{
Alexander V. Gerasimov ${ }^{1}$, Roman O. Cherepanov ${ }^{1}$, Raisa A. Krektuleva ${ }^{2 *}$ and Vladimir $\mathrm{N}$. \\ Barashkov $^{3}$ \\ ${ }^{1}$ National Research Tomsk State University, 634050 Tomsk, Russia \\ ${ }^{2}$ National Research Tomsk Polytechnic University, 634050 Tomsk, Russia \\ ${ }^{3}$ Tomsk State University of Architecture and Building, 634050 Tomsk, Russia
}

\begin{abstract}
Heat and mass transfer during absorption on a film of lithium bromide water solution flowing by a cooled wall in the steam atmosphere is numerically investigated in this paper. The self-similar solutions are using as the initial conditions for solving the problem beyond the entrance region. The key criteria characterizing heat and mass transfer in the film absorption with uniform velocity profile and with a constant thickness have been determined.
\end{abstract}

\section{Problem statement}

Numerical simulation of gas-thermal spray coatings is a difficult task, the urgency of which is due to the widespread application of technologies for the deposition of hardening coatings. The interaction of sprayed particles with the surface of a processed material is three-dimensional, has a wide range of impact velocities (from 100 to $2000 \mathrm{~m} / \mathrm{s}$ ) and a high strain degree. The melted particles interacting with the surface can contain solid inclusions, which makes it difficult to simulate the behavior of this media.

This paper represents the simulation results of melted viscous metal drops impacting with a cold surface, as well as the crystallization process using the SPH method.

\section{Computational method}

The paper considers a variant of the SPH method, namely a symmetrized method of smoothed particles. The SPH approximation of the unsteady heat transfer equations is used to calculate thermal conductivity.

The symmetrized SPH method is conservative concerning the momentum, almost conservative concerning the energy, has a first-order time accuracy, but it has a low approximation accuracy of spatial derivatives (of order zero in the general case). The method allows free boundary problems to be solved.

The method is based on nuclear approximation:

\footnotetext{
* Corresponding author: $\underline{\text { rakrekt@ } @ \text { tpu.ru }}$
} 


$$
f(\vec{x}) \cong \int f(\vec{y}) W(\vec{y}-\vec{x}, h) d \vec{y}
$$

where $\vec{x}, \vec{y}$ is the radius vectors (spatial coordinates), $\mathrm{W}$ is the smoothing function (usually a B-spline) [1], $\mathrm{h}$ is the smoothing parameter that determines the area of support for the smoothing function. The approximation (1) of partial spatial derivatives is given by:

$$
f(\vec{x})_{, \alpha}=\frac{\partial f(\vec{x})}{\partial x_{\alpha}} \cong \int f(\vec{y}) W(\vec{y}-\vec{x}, h)_{, \alpha} d \vec{y}
$$

The discrete form of the approximation (2) has the form:

$$
f_{, \alpha}^{n}=\sum_{k} f^{k} \frac{m^{k}}{\rho^{k}} W_{, \alpha}^{n k}
$$

The equations of the viscous compressible fluid motion have the form:

$$
\begin{gathered}
\rho \dot{v}_{i}=-\sigma_{i j, j}, \\
\sigma_{i j}=-P \delta_{i j}+\mu \dot{\varepsilon}_{i j}, \\
P=P(\rho, E), \\
\dot{\varepsilon}_{i j}=\left(v_{i, j}+v_{j, i}\right) / 2 ;
\end{gathered}
$$

where $\rho$ is the density of the medium, $v_{i}$ is the components of the velocity vector, $\sigma_{i j}$ is the stress tensor components, $P$ is the pressure, $\mu$ is the viscosity of the medium, $E$ is the internal energy.

The energy balance equation is given in the form:

$$
\dot{E}=\sigma_{i j} \dot{\varepsilon}_{i j}+\left(\lambda T_{, j}\right)_{, j}
$$

The temperature is considered to be an internal energy function:

$$
\dot{T}=\dot{E} /\left(C_{P} \rho\right)
$$

The approximation of equation (4) with the use of (3) does not meet the law of conservation of momentum and energy (nonconservative concerning the momentum and energy). To restore conservatism, a transformed form of the equation (4) is used:

$$
\rho \dot{v}_{i}=-\left(\sigma_{i j, j}+\left[1_{, j}\right] \cdot \sigma_{i j}\right)
$$

In (10) the gradient of one (mathematically equal to zero) is used to adjust the SPH approximation of the stress divergence and make it symmetrical [2], thus ensuring the equality of forces acting between a pair of SPH nodes. After applying the SPHapproximation (3) to (10) we have:

$$
\dot{v}_{i}^{n}=\frac{1}{m^{n}} \sum_{k} \frac{m^{n} m^{k}}{\rho^{n} \rho^{k}}\left(\sigma_{i j}^{k}+\sigma_{i j}^{n}\right) W_{, j}^{n k}
$$


To calculate the heat transfer, the heat flow divergence is calculated in a similar manner:

$$
\begin{gathered}
\dot{T}^{n}=\frac{1}{C_{P}^{n}}\left[\frac{\sigma_{i j}^{n} \varepsilon_{i j}^{n}}{\rho^{n}}+\frac{1}{m^{n}} \sum_{k} \frac{m^{n} m^{k}}{\rho^{n} \rho^{k}}\left(q_{i}^{k}+q_{i}^{n}\right) W_{, i}^{n k}\right] \\
q_{i}^{n}=\lambda^{n} \sum_{k} \frac{m^{k}}{\rho^{k}} T^{k} W_{, i}^{n k}
\end{gathered}
$$

In both cases, the symmetrization procedure (10) allows the energy conservation to be (almost) achieved, that is the calculated acceleration and strain rate values meet the law of conservation of energy:

$$
\sum_{k} m^{k} v_{i}^{k} a_{i}^{k}=\sum_{k} \sigma_{i j}^{k} \dot{\varepsilon}_{i j}^{k} V^{k}
$$

However, the total conservatism of the scheme is broken by integrating the equations of motion in time, since the error is accumulated per the time step when calculating the kinetic energy component:

$$
\Delta E_{K}=\sum_{k} \Delta t \cdot m^{k} v_{i}^{k} a_{i}^{k}+\sum_{k} \frac{\Delta t^{2}}{2} m^{k} a_{i}^{k} a_{i}^{k}
$$

The second term in (14) introduces an error in the calculation of the total energy balance. The magnitude of this error was controlled in the computations; the total losses during the transition of kinetic energy into potential one did not exceed $4 \%$ in the computations at initial moments of time after the impact at the initial velocity of particles up to $2000 \mathrm{~m} / \mathrm{s}$.

The Stefan problem was solved by the shock-capturing method. For this purpose, the thermal energy of each computational point was calculated at each time step. When achieving the melting temperature, the temperature growth was stopped, and the internal energy was accumulated to reach the value of the latent melting heat, after which the material was considered to be completely melted and was calculated using a model of an ideal viscous compressible fluid. If the internal energy dropped below the melting energy, the material was considered to be solid and was calculated by the model of an ideal elasticplastic body.

The scheme described was used to simulate the interaction of melted metal drops with a cold surface.

The impact of a melted metal drop with an iron substrate was considered to be a test problem, and the metal parameters were taken for the $36 \mathrm{NHTU}$ steel from the reference book [3]: the density was $7.87 \mathrm{~g} / \mathrm{cm}^{3}$, the melt viscosity was $5.4 \mathrm{mPa} / \mathrm{s}$, T of melting was $1280 \mathrm{~K}$, Q of melting was $84 \mathrm{~J} / \mathrm{g}$, the heat capacity was $0.56 \mathrm{~J} / \mathrm{g}, \lambda=28 \mathrm{~W} /(\mathrm{m} \mathrm{K})$; the elastic metal properties were constant and did not depend on the temperature for a first approximation: $\mathrm{E}=200 \mathrm{GPa}, \mathrm{G}=70 \mathrm{GPa}, \mathrm{Y}_{0 \text {,dynamic }}=1.2 \mathrm{GPa}$.

The computations were performed for three impact velocities: 100,150 and $200 \mathrm{~m} / \mathrm{s}$, the initial temperature of the drop was $1380 \mathrm{~K}$, and the diameter of the drop was 8 microns. The process was computed until the maximum particle velocity dropped up to $50 \mathrm{~m} / \mathrm{s}$. The cross sections of the drops are shown in Figure 1 for the final stages of impact. 


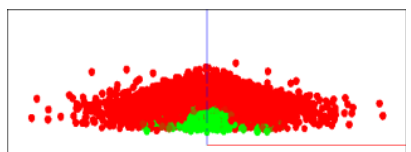

a)

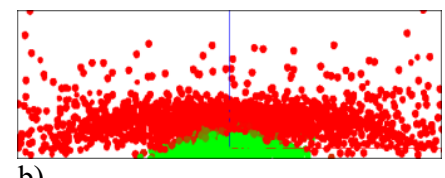

b)

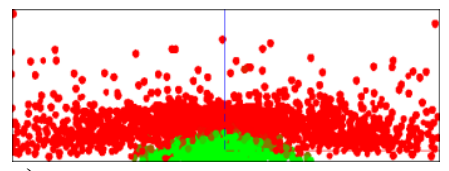

c)

Fig. 1. Cross sections of drops at the final stages of the process, (a) $100 \mathrm{~m} / \mathrm{s}$, (b) $150 \mathrm{~m} / \mathrm{s}$, (c) $200 \mathrm{~m} / \mathrm{s}$, the red color denotes the melt, the green color denotes the crystallized material.

\section{Conclusion}

The computations obtained by the model described allowed us to find that the impact velocity of about $150 \mathrm{~m} / \mathrm{s}$ was critical. At lower velocities the major part of the drop maintains uniformity and, after cooling, a larger portion of the drop mass forms a hardening coating on the surface. At higher velocities the drop is significantly failed and forms a cloud of disintegrated material, and less than $20 \%$ of the original melt mass is crystallized on the surface. The disadvantage of this model is that the surface tension forces are not considered, and therefore these computations are very approximate and can characterize the process only qualitatively.

The study was funded by the Russian Science Foundation (project No.16-19-10264).

\section{References}

1. L.B. Lucy. Astron. J. 82, 1013 (1977)

2. Shaofan Li, Wing Kam Liu, Meshfree Particle Methods, (Springer Science \& Business Media, Germany , 2007)

3. I.K. Kikoin, Table of Physical Quantities. Reference Book (Atomizdat, Moscow, 1976) 